\title{
Gastric Cancer:Risk Factors, Diagnosis and Management
}

\author{
Murtaza Mustafa ${ }^{1}$, Jayaram Menon ${ }^{2}$, RK.Muniandy ${ }^{3}$, EM.Illzam ${ }^{4}$,

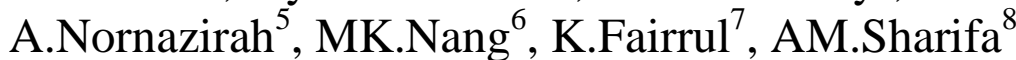 \\ ${ }^{1,3,5,6,7}$, Faculty of Medicine and Health Sciences, University Sabah, Malaysia, Kota Kinabalu, Sabah, Malaysia. \\ 2. Department of Gastroenterology, Hospital Queen Elizabeth,Kota Kinabalu, Sabah, Malaysia \\ 3.Clinic Family Planning Association, Kota Kinabalu,Sabah,Malaysia \\ 4. Quality Unit Hospital Queen Elizabeth,KotaKinabalu,Sabah,Malaysia.
}

\begin{abstract}
Gastric cancer $(G C)$ is the most common cancer with high mortality worldwide. Highrate of GC deaths reported in China, Japan, and Korea. There are 56\% new cases from Eastern Asia,41\% from China, and11\% from Japan. The common causes of GC include Helicobacter pylori infection which accounts for $60 \%$ of cases, pickled vegetables, smoking, alcohol intake and genetics. GC is often asymptomatic or nonspecific symptoms. Early symptoms are associated with indigestion, heartburn, abdominal discomfort, loss of appetite, nausea, vomiting, diarrhea or constipation, blood in the stool (melena], and weight loss. Patient's history and endoscopic examination is the diagnostic method. Cancer staging, and TNM (tumor, node, and metastasis),systems is diagnostic. Treatment for GC may include surgery, chemotherapy and/ or radiotherapy. New treatment approaches such as biological therapy combined with current methods are being studied in clinical trials. Prognosis of GC is generally poor due to metastasis and advanced age at presentation. Eradication of H.pylori infection, a healthy diet high in fruits and vegetables was associated with lower risk of $G C$.
\end{abstract}

Keywords: Gastric cancer, Helicobacter pylori, Diagnosis, Management.

\section{Introduction}

Gastric cancer(GC) or cancer or, is cancer developing from the inner lining of the stomach[1].The cancer may spread from the stomach to other parts of the body, particularly liver, lung, bones, lining of abdomen and lymph nodes [2],Worldwide, stomach cancer is the fifth most common cancer with 952,000 cases diagnosed in 2012 and 723,000 deaths[3]It is more common in men and in developing countries[4].It is the third leading cause of cancer death after lung cancer and liver cancer[5].Less than 5\% of stomach cancers occur in people under 40 years of age and $81.1 \%$ of that $5 \%$ in the age-group of 30 to 39 and $18.9 \%$ in the age group of 20 to 29 [6].In 2014,stomach cancer accounted for $0.61 \%$ of deaths(13,303 in the United States)[7].In China,stomach cancer accounted for $3.56 \%$ of all deaths $(324,438)[8]$.The highest rate of stomach cancer was in Magnolia,at 28 cases per 100,000 people[9].There are 56\% of new cases from Eastern Asia,41\% from China,and $11 \%$ from Japan[10].In Thailand, the incidence rate of gastric cancer was 4.1:100,000 for males and $2 .: 10,000$ in females[11].In Malaysia, the incidence rate of gastric cancer was $4.3 \%$ in males.[12].The most common cause is infection by the bacterium Helicobacter pylori, which accounts for $60 \%$ of cases[13].Other common causes include eating picked vegetables and smoking. Genetic and smoking also plays an important role[14].Early symptoms include heartburn, upper abdominal pain, nausea and loss of appetite, difficultyswallowing, and blood in the stool. [2].Diagnosis is by usually by biopsy done during endoscopy, followed by medical imaging[2].Treatments may include some combination of surgery, chemotherapy, radiation therapy, and targeted therapy[2].Outcomes are often poor with less than 10\% 5-year survival rate globally[15].Mediterranean diet lowers the risk of cancer as does stopping of smoking. There is tentative evidence that treating $H$.pylori decreases the future risk[14].The paper reviews the current literature, the association of H.pylori infection in gastric cancer along with diagnosis and management of gastric cancer.

\section{Risk factors}

Gastric cancer occurs as a result of many contributory factors or causes [16].It occurs twice as common in males than females-estrogen may protect women against the development of this from[17].

Helicobacter pylori infection:Helicobacter pylori was identified in 1982 by two Australian scientists Robin Warren and Barry J Marshal as a causative factor for ulcer[18].In developing countries, where most children become infected by the age 10 of gastric cancer rates are high[19].H.pylori is able to survive and multiply in gastric environment, which is hostile to the growth of other bacteria[20].Numerous adaptations permit survival of H.pylori in the acidic milieu of the stomach[21].Although most organisms appear to be adherent to the mucosal epithelial cells and form adherence pedestal resembling those produced by enteropathogenicEscherichia coli, several important adhesions have been identified[22].All H.pylori spp. causes 
some degree of persistent inflammation in the mammalian stomach. Gastritis is found in virtually all infected humans, although majority has no symptoms; only one in 10 develop ulcer disease. Gastric adenocarcinoma is 3 to 12 times more likely to develop in individuals infected with H.pylori [23].H.pylori is more likely to associated with the early or initial states of primary gastric lymphoma than advanced tumors;H.pylori can disappear during progression of gastric lymphoma [24].Eradication of H.pylori is associated with significant reduction in duodenal ulcer recurrence, and is also useful in differentiating between H.pylori gastritis and gastric MALT, lymphoma(mucosa-associated lymphoid tissue)[25,26].In H.pylori infected patients who develop gastric cancer, serum IgG against $\mathrm{CagA}$ is $94 \%$ sensitive and $\mathrm{p} 3 \%$ specific, indicating that detection of antibodies to CagA is useful marker for diagnosis of duodenal and gastric cancer[27].

H.pylori is an essential risk factor in $60-80 \%$ of gastric cancers, but only $2 \%$ of people with Helicobacter pylori infections develop stomach cancer[28].The mechanism by which H.pylori induces stomach cancer potentially involves chronic inflammation, or the action of H.pylorivirulence factors such as CagA[29].Other studies advocate that H.pylori infection is widely regarded as the most important risk factor in the development of gastric cancer(GC)[30],with $0.5 \%$ to $2 . \%$ developing gastric adenocarcinoma[31].A metaanalysis of 34 cohort and case-control studied patients found that H.pylori carried a relative risk of GC of $3.02(95 \%$ CI:1.92-4.74) in high risk settings(china,Japan and Korea)and 2.56(95\%CI:1.99-3.39) in low risk settings(Western Europe, Australia, and the United States[32].Epidemiological data indicates that GC occurs more frequently in populations with higher rates of H.pyloriinfection, and the World Health Organization has classified this bacterium as a Class 1 carcinogen for GC[28].H.pylori infection was important in the process of tissue remodeling, angiogenesis, tumor invasion and metastasis[33]and induces a number of genes in the host cells that are potential determinants of inflammation, angiogenesis, and metastasis including interlukin-8(IL8)gene expression[29].However, it remains unclear howH.pylori infection activates specific transcription factors and induces gene expression [34]..Yamada et al.indicated that H.pylori in in Thai GC patients was reported by combined histopathology and H.pylori $\operatorname{IgG}$ antibody test with $77.1 \%$ and $97.4 \%$ of sensitivity and specificity, respectively[35].Moreover,IL-8mRNA expression is one of the factors that were possible influences which affect GC[36].Yamada et al.reported that GCs were detected in more than $80 \%$ of Thai patients with high levels of IL-8 mRNA expression, whileH.pylori infection and IL-8 mRNA expression were relative risks for Thai GC,therefore IL-8 mRNA expression may be useful diagnostic and prognostic risk marker for GC[25].Similarly Marci et al. indicated that the level of serum IL-8 mRNA expression may act as marker of GC[37].The high expression of IL-8 mRNA expression was directly demonstrated with a poor prognostic histologic type in GC[35].It was estimated that Epstein-Barr virus is responsible for 84,000 cases per year [38].Other factors associated with increased risk are AIDS[28].

Smoking and Alcohol: Smoking increases the risk of developing gastric cancer significantly, from $40 \%$ increased risk for current smokers to $82 \%$ increased risk for heavy smokers. Gastric cancers due to smoking mostly occur in the upper part of the stomach near the esophagus[39].Some studies show risk with alcohol consumption as well[28].Consumption of alcohol and smoking are risk factors. Chronic alcohol disturbs gastric mucosal barrier by inhibiting COX 1 receptor enzymes which reduce the production of cytoprotectiveprostaglandin.Cigarette smoking causes reduction of circulating epidermal growth factor and increase free radical production in gastric mucosa[40,41].Researchers in Denmark in a series of 2416 subjects found that tobacco smoking and H.pylori infection are the main risk factors for peptic ulcer disease(PUD) in Danish adults[42].Studies have found that alcohol consumption increases risk when associated with H.pylori infection[43].Satarasinghe and colleagues in a series of 1500 patients found alcohol was a contributory factor in one third of gastrointestinal bleeding(GIB) patients[44].

Diet and gastric cancer: Dietary factors are not proven causes but some foods including smoked foods[45,46],salt and salt-rich foods, red meat, processed meat, pickled vegetables[28,47], and bracken are associated with a higher risk of stomach cancer. Nitrates and nitrites in cured meats can be converted by certain bacteria, includingH.pylori, into compounds that have been found to cause stomach cancer in animals [47].

Fresh fruits and vegetables intake, citrus fruit intake, and antioxidant intake are associated with lower risk of stomach cancer [28].A Mediterranean diet is associated with lower rates of stomach cancer[48].Buckland and associated in a series of 485,044 subjects (144,577 men) aged 35-70 year from 10 European countries, concluded that greater adherence to an Mediterranean diet is associated with a significant reduction in the risk of incident gastric cancer[48].Regular aspirin use is also associated with lower risk of gastric cancer[28].Intake of total fat is positively associated with gastric cancer risk, and specific subtypes of fats account for different effects[49].

Obesity is a physical risk factor that has been found to increase the risk of gastric adenocarcinoma by contributing to the development of gastroesophageal reflux disease (GERD)[50].The exact mechanism by which obesity causes GERD is not completely known. Studies hypothesize that increased dietary fat leading to increased pressure on the stomach and the lower esophageal sphincter, due to access adipose tissue, could play a role,yet no statistically significant data has been collected[51].However, the risk of gastric cardia 
adenocarcinoma, with GERD present has been found to increases more than 2 times for an obese person[50].There is a correlation between iodine deficiency and gastric cancer[52].

Nagel and associates in a series of 520,000 participants, concluded that higher socioeconomic position was associated with reduced risk of gastric adenocarcinoma[53].Higher HDI( highest human development index)values, coupled with higher prevalence of male obesity and a higher per capita consumption of alcohol, tobacco, fruits vegetables and meat are associated with a higher incidence of gastric cancer based on an analysis of populational global data[54].

Role of genetics and family history:About $10 \%$ of cases run in families and between $1 \%$ and $3 \%$ of cases are due to genetic syndromes inherited from person's parents such as hereditary diffuse gastric cancer[14].A genetic risk factor for gastric cancer is a genetic defect of the CDHI gene known as hereditary diffuse gastric cancer(HDGC).The CDHI gene, which codes for E-cadherin,lies on the $16^{\text {th }}$ chromosome[55].. When the gene experiences a particular mutation,gastric cancer develops through a mechanism that is not fully understood[55].The mutation is considered autosomal dominant meaning that half of a carrier's children will likely experience the same mutation[55].Diagnosis of hereditary diffuse gastric cancer usually takes place when at least two cases involving a family member, such as parent or grandparent, anddiagnosed, with at least one diagnosed before the age of 50[55].The diagnosis can also be made if there are at least three cases in the family, in which case age is not considered[55].International Cancer Genome Constorium is leading efforts to identify genomic changes involved in stomach cancer[56].A very small percentage of diffuse-type cancers arise from an inherited abnormal CDHI gene.Genetic testing and treatment options are available for families at risk[57].

Miscellaneous risk factors include diabetes,perniciousanemia,chronic atrophic gastritis[5860],Menetrier's disease(hyperplastic,hypersecretorygastropathy),and intestinal metaplasia[61,62].

\section{Clinical Manifestations}

Stomach cancer is often either asymptomatic (producing no noticeable symptoms) or it may cause only nonspecific symptoms (symptoms that specific to stomach cancer and to other related or unrelated disorders).By the symptoms occur, the cancer has often reached and advanced stage and may have metastasized (spread to other, perhaps distant, part of the body), which is one of the reasons for its relatively poor prognosis[63].Early symptoms of gastric cancer includes:[64].

a).Early cancers may be associated with indigestion or burning sensation (heartburn).However, less than 1 in 50 people referred for endoscopy due to indigestion has cancer.

b).Abdominal discomfort and loss of appetite, especially for meat may occur.

c).Gastric cancer that have enlarged and invaded normal tissue can cause weakness, fatigue, bloating of stomach after meals, abdominal pain in the upper abdomen, nausea and occasional vomiting, diarrhea or constipation.

d).Further enlargement may cause weight loss or bleeding with vomiting blood or having blood in the stool. The latter apparent as black discoloration (melena) and sometimes leading to anemia. Dysphagia suggests a tumor in the cardia or extension of the gastric tumor into the esophagus.

e).These can be symptoms of other problems such as a stomach virus,gastric ulcer, or tropical sprue.

\section{Diagnosis}

Patient's history and gastroscopic examination is the diagnostic method [59].Upper GI series (barium roentgenogram), and computed tomography or CT scanning of the abdomen may revel gastric cancer[65].In 2013, Chinese and Israeli scientists reported a successful pilot study of a breathalyzer-style breath test indented to diagnose stomach cancer by analyzing exhaled chemicals without the need for an intrusive endoscopy [66].A biopsy with subsequent histological analysis, is the only sure way to confirm the presence of cancer cells[59].Other gastroscopic modalities such as optical coherence tomography are being tested investigationally for similar applications[67].A number of cutaneous conditions are associated with gastric cancer include tripe palms, a darkening hyperplasia of the skin of palms[68].Blood tests for tumor markers, such as $\mathrm{CEA}$ (carcinoembryonic antigen) and $\mathrm{CA}$ ( carbohydrate antigen) may be ordered, as their levels correlate to extent of metastasis especially the liver, and the cure rate[69].

Gastric cancer staging: The clinical staging of gastric cancer includes:[70,71].

Stage 0.Limited to the inner lining of the stomach.Treatable by endoscopic mucosal resection otherwise by gastrectomy and lymphadenectomy without need for chemotherapy or radiation.

Stage 1.Pnenetration to second or third layers of stomach (Stage 1A) or to the second layer and nearby lymph nodes (Stage 1B).Stage IA and Stage IB is treated by surgery and chemotherapy respectively. Stage IB may be treated with chemotherapy (5-flurourocil) and radiation therapy.

Stage 11.Penetration to the second layer and more distant lymph nodes. Treated as for Stage1, sometimes with additional neoadjuvant chemotherapy. 
Stage 111.Pnetration to the third layer and more distant lymph nodes, or penetration to the fouth layer or more distant lymph nodes. Treated as for Stage 11; a cure is still possible in some cases.

Stage IV.Cancer has spread to nearby tissues and more distant lymph nodes or has metastastasized to other organs. A cure is rarely possible at this stage.The TNM (tumor,node,and metastasis) system is also used[72].In a Thai study, tissue samples were taken by endoscopy with tissue $I L-8 m R N A$ expression conducted by real time relative quantitation polymerase chain reaction[73].

\section{Management}

Cancer of stomach is difficult to cure unless it is found at an early stage. The disease is usually advanced when diagnosis is made [74].Treatment for gastric cancer may include surgery, chemotherapy, and / or radiation therapy[75,76].New treatment approaches such as biological therapy and improved ways of using current methods are being studied in clinical trials[77].

Surgery: Surgery remains the only curative therapy for stomach cancer [15].0f different surgical techniques, endoscopic mucosal resection(EMR) is a treatment for early cancer(tumor only involves the mucosa) that was pioneered in Japan and is available in the United States at some centers[15].In this procedure, the tumor, together with the inner lining of stomach(mucosa), is removed from the wall of the stomach using an electrical wire loop through the endoscope. The advantage is that it is a smaller operation than removing the stomach[15].Endoscopic submucosal dissection(ESD) is a similar technique pioneered in Japan used to resect a large area of mucosa in one piece[15].If the pathologic examination of resected specimen shows incomplete resection or deep invasion by tumor, the patient would need a formal stomach resection[15].A 2016 Cochrane review found low quality evidence of no difference in short-term mortality between laparoscopic and open gastrectomy(removal of stomach), and that benefits or harms of laparoscopic gastrectomy cannot be ruled out[78].Those with metastatic disease at the time of presentation may receive palliative surgery and while it remains controversial, due to the possibility of complications from surgery itself and the fact that it may delay chemotherapy the data so far is mostly positive, with improved survival rates being seen in those treated with this approach[16,79].

Chemotherapy, Targeted and Radiotherapy:The use of chemotherapy to treat stomach cancer has no firmly established standard care.Unfortunately, stomach cancer has not been particularly sensitive to these drugs,and chemotherapy, if used, has usually served to palliatively reduce the size of tumor, relieve symptoms of the disease and increase survival time.Some drugs used in stomach cancer treatment have included 5$\mathrm{FU}($ fluorouracil) or its analog capecitabine,BCNU(Carmustine),methyl-CCNU(semustine) and doxorubicin (Adriamycin),as well as mitomycin $\mathrm{C}$, and more recently cisplatin and taxotere, often using drugs in various combinations. The Relative benefits of these drugs, alone and in combination, are unclear[80].Clinical researchers have explored the benefits of giving chemotherapy before surgery to shrink the tumor,or as adjuvant therapy after surgery to destroy remaining cancer cells[15].

Recently, treatment with human epidermal growth factor receptor 2(HER2) inhibitor, trastuzumab,has been demonstrated to increase overall survival in inoperable locally advanced or metastatic gastric carcinoma over-expressing the HER2/neu gene[15].In particular,HER2 is overexpressed in 13-22\% of patients with gastric cancer [77, 81].0f note,HER2 overexpression in gastric neoplasia is heterogeneous and comprises a minority of tumor cells (less than $10 \%$ of gastric cancers overexpress HER2 in more than 5\% of tumor cells). Hence, this heterogeneous expression should be taken into account for HER2 testing, particularly in small samples such as biopsies, requiring the evaluation of more than one biopsy sample[81].Radiotherapy may be used to treat stomach cancer, often as an adjuvant to chemotherapy and/or surgery[15].

\section{Prognosis and Prevention}

The prognosis of gastric cancer is generally poor, due to the fact the tumor has often metastasized by the time of discovery and the fact that most people with the condition are elderly (median age is between 70 and 75 years) at presentation [82].The five years survival rate for gastric cancer is reported to be less than 10 percent[15].

Prevention:Getting rid of H.pylori in those who are infected decreases the risk of gastric cancer,at least in those who are Asian[83].A 2014 meta-analysis of observational studies found that a diet high in fruits, mushrooms, garlic, soybeans, and green onions was associated with a lower risk of stomach cancer in Korean population [84].Low doses of vitamins, especially from a healthy diet, decreases the risk of stomach cancer[85].A previous review of antioxidant supplementation did not find supporting evidence and possibly worse outcomes[86]. 


\section{Conclusions}

Helicobacter pylori infection was associated with risk of gastric, duodenal ulcers and gastric cancer. The eradication of H.pylori in those who are infected decreases the risk of gastric cancer. Despite the advancement of medical diagnosis prognosis of gastric cancer is generally poor.

[1]. Stomach (gastric)Cancer.NCI.Retrived 1 July 2014

\section{References}

[2]. Ruddon Raymond W.(2007).Cancer biology(4 ${ }^{\text {th }}$ Ed.)Oxford University Press.p.223.ISBN9780195431.

[3]. Chapter 1.1.World Cancer Report 2014.World Health Organization.2014.ISBN 9283204298.

[4]. ParkinDM,BrayF,FerlayJ,etal.Global Cancer Statistics,2002.CA:A Cancer J Clin.2005;55(2):74-108.

[5]. Lozano R,NaghaviM,ForemanK,etal.Global and regional mortality from 235 causes of death for 20 age groups in 1990 and $2010:$ a systematic analysis for Global Burden of Disease Study 2010.Lancet.2012;380(9859)2095-128.

[6]. Gastric Cancer in Young Adults.RevistaBrasileira de Cancerrolgia.46(3).July 2000,

[7]. Health Profile:UnitedStates.LeDucMedia.Retrieved 31 January 2016.

[8]. Health Profile:China.LeDucMedia.Retrieved 31 Jan 2016,

[9]. Stonach Cancer: Death Rate Per 10,000.Le DucMedia.Retrieved 13 March 2014.

[10]. Inoue M,TsuganeS.Epidemiology of gastric cancer in Japan.Posgrad Med J.2005;81:419-424.

[11]. KhuhapremaT,SrivatanakulP.Cancer in Thailand Vol IV Bangkok:Bangkok Medical Publisher,2007;32-33.

[12]. National Cancer Registry Report.Malaysian Cancer Statistics-Data and Figure.2007.

[13]. Sim Edited by Fiona,McKee Martin(2011).Issues In Public Health(2 ${ }^{\text {nd }}$ Ed.) Maidenhead:Open University Press.p.74.ISBN 9780335244225 .

[14]. World Cancer Report 2014.World Health Organization.2014.pp.Chapter 5.4.ISBN 9283204298.

[15]. OrdithuraM,GaliziaG,SforzaV,etal. Treatment of Gastric Cancer.World J Gastrol.2014;20(7):1635-49.

[16]. Lee YY,DerakhshanMH.Environmental and lifestyle risk factors of gastric cancer.Arch Iran Med.2013;16(6):2397-403.

[17]. ChandannosE,Lagergren J,0estrogen and enigmatic male predominance of gastric cancer.Eur J Cancer.2008;44(16):2397-403.

[18]. Marshall BJ.Unidendified Curved Bacillus On Gastric Epithelium In Active Chronic Gastritis.Lancet.1983;(8336):1273-75.

[19]. Telford JL,Covacci A, GhiaraP,etal.Unravelling Role of Helicobacter pylori in Peptic ulcer:Potential New Therapies and Vaccines.Trend Biochem.1994;12:420-6.

[20]. Marshal BJ,WarrenJR.Unidentified Curved Bacilli In the Stomach of Patients with Gastritis and Peptic Ulceration.Lancet.1.1984;(8390):131-15.

[21]. Ulcer Diagnosis And Treatment CDC Bacterial Mycotic Diseases (Http: www.cdc.Gov/Ulcer/History,Htm).2014.

[22]. JiverD,ArnqvistA,PgrenJ,etal.Helicobacter pyloriAdhesin Binding FucosylatedHisto-Blood Group Antigens Revealed by Retagging.Science.1998;279:373-77

[23]. Tompkins JS,FallowS.The Path To Preventing Unlcers.Science. 1995;267:1621-2..

[24]. Nakamura S,YaoT,Aoyagi K, et al.Helicobacter pylori And Primary Gastric Lymphoma A Holistic andImmunohistological Analysis of 237 Patients.Cancer.1977;79:3-11.

[25]. PatchettS,Beaties S, LeenF,etal.Heliocobacter pylori And Duodenal Ulcer Recurrence.Am J Gastroenterol.1992;87:24-7.

[26]. Stolte M, Helicobacter pylori Gastritis And Gastric MALT-Lymphoma (Letter). Lancet.1992;339:745-6.

[27]. BlaserMJ,Perez-Perez GL, KleanhouseHK,etal.Infection with Heliocobacter pylori Strains posessinhCaga is associated With An increased risk Developing Adenocarcinoma of the Stomach.Cancer Res.19995;55:2111-15.

[28]. Gonzalez CA,SalaN,RokkasT,etal.Gastriccancer:epidemiologic aspects. Helicobacter.2013;18(Supplement 1):34-38.

[29]. Hatakeyama M, Higashi H; Higashi.Helicobacter pylori Cag A:a new paradigm for bacterial carcinogenesis.CancerScience.2005;96(12):835-43.

[30]. Lee KE.KoiPN,XiaY,etal.Helicobacter pylori and interleukin-8 in gastric cancer.World J Gasteroenterol.2013;19:8192-8202.

[31]. Atherton JC,The pathogenesis of Helicobacter pylori-induced gastro-duodenal diseases.Annu Rev Pathol.2006;1:63-97.

[32]. Cavalerio-pinto M,PeleterioB,LunetN,etal.Helicobacter pylori infection and gastric cardia cancer:systematic review and metaanalysis.Cancer Causes Control.2011;22:375-87.

[33]. Iwamoto J,MizokamiY,TakahshiK,et al. Expressions of urokinase-type plasminogen activator,its receptor and plasminogen activator inhibitor-1 in gastric cancer cells and effects of Helicobacter pylori.Scand J Gasteroenterol.2005;40:783-93.

[34]. FutagamiS,HiratsukaT,TatsuguchiA,etal.Monocyte chemoattractant protein 1(MCP) released from Helicobacter pylori stimulated gastric epithelial cells includes cyclooxygenase 2 expression and activation in T cells.Gut.2003;52:1257-64.

[35]. Yamada S,KatoS,MatsuhisaT, et al. Predominant muosal IL-8 mRNA expression in non-cagA Thais is risk for gastric cancer.WorldJ Gastroenterol.2013;19:2941-49.

[36]. KozlovSV.Inflammaton and cancer Methods and protocols.Volume !:Experimental models and practical approaches.Preface,MethodsMol Biol.2008;511:v-viii.

[37]. Marci A.VersaciA,LoddoS,etal.Serum levels of interleukin 1beta,interleukin 8 and yumor necrosis factors alpha as markers of gastric cancer.Biomarkers.2006;11:184-193.

[38]. http;//www.cancerresearchuk.org/about-us/cancer-news/press-release/2014-03-24-developing-a-vaccine- for-the-epstein-barr-viruscould-prevent-up-to-200000-cancers - globally -say.

[39]. What Are the Risk Factors for Stomach Cancer (Website).American Cancer Society?Retrieved 2010-03-31.

[40]. KoJK,ChoCH,Alcohol drinking and cigarettesmoking A Partner for Gastric Ulceration.Zhomghua Yi XueZa Zi.2000;63(12):85454.

[41]. Mal Chow JY,ChoCh.Effect of Cigarette Smoking on Gastric Ulcer Formation and Healing Mechanism of Action.ClinGasteroenterol.1998;S80-6.

[42]. RosenstockS,JergersenT,Bonnevie 0,et al.Risk Factors for Peptic Ulcer Disease. A Population Based Prospective Study Comp[rising 2416 Danish Adults.Gut.2003;52:186-93.

[43]. SalihBarik,FaithAbasiyanik,NizamettinBayyurt,etal.H Pylori Infection and Other Risk Factors Associated With Peptic Ulcer in Turkish Patients:A retrospective Study.World J Gasteroenterol.2007;13(23):32554-8.

[44]. SatarasingheRL,De Silva AP,ArulnithyK,etal.Aetiology And Other Features of Adult Sri Lankan Presenting With Upper Gastrointestinal Bleeding(UGIB).J Ceylon College Physic.2010;41:57-60.

[45]. Tumors of the GI Tract(http://www.merck.com/mmpe/sec021/Ch021/cho21d.html)at Merck Manual of Diagnosis and Therapy Profession Edition. 
[46]. JakszynP,Gonzalez CA Gonzalez.Nitrosomine and related food intake and gastric and oesophageal cancer risk:A systematic review of the epidemiological evidence (PDF).World J Gastroenterol.2006;12(2\&):4296-4303.

[47]. Alonso-AmelotME,Avendano M, Avendano.Human Carcinogenesis and bracken fern:a review of evidence.Curr MedChemist.2002;9(6):675-86.

[48]. Buckland G,Agudo A, Lujan L,etal.Adherence to Mediterranean diet and risk of gastric adenocarcinoma within European Prospective investigation into Cancer and Nutrition(EPIC) cohort study.Am J Clin Nutri.2009;91(2):381-90.

[49]. Jun Han,YiJang,XiaoLiu,etal.Dietary Fat Intake and Risk of Gastric Cancer:A Meta -Analysis of Observational Studies. (http: //journals. plos.org/ plos. org/ plosone/article?id=10.1371/journal.pone.0138580.

[50]. Crew K,NeugutA.Epidemiologu of gastric cancer.Worl J Gastroenterol. 2006; 12 (3):354-62.

[51]. HampelHoward,AbrahamNeenaS,El-Serag Hashem B.Meta-analysis:obesity and the risk of gastroesophageal reflux and its complications.Ann Intern Med. 2005; 143(3):199-211.

[52]. JosefsssonM,Ekbld E.22 Sodium Iodide Symporter(NIS) in Gastric Mucosa:Gastric Iodide Secretion.InPreedy Victor R;Burrow Gerard N;Watson Ronald. Comprehensive Handbook of Iodine Nutritional,Biochemical Pathological and Therapeutic Aspects.Elsevier.pp.215220.ISBN 978-0-12-374135-6.

[53]. Nagel G,LinseisenJ,BoshuizenHC,etal.Scocioeconomic position and the risk of gastric and esophageal cancer in the European Prospective Investigation into Cancer and Nutrition(EPIC-EURGAST).Int J Epidemiol.2007;36(1):66-76.

[54]. Ferrari F,ReisMA.Study of risk factors for gastric cancer by population databases analysis.World $J$ Gastroenterol.2013;19(48):9383-91.

[55]. Hereditary Diffuse Cancer .No Stomach for Cancer. Retrieved 21 Oct 2014

[56]. Gastric Cancer-Adenocarcinoma.Int Cancer Genome Consortium.Retrieved 24 February 2014.

[57]. Brooks-Wilson AR,KaurahP,etal.Germline E-cadherin mutations in hereditary diffuse gastric cancer:assessment of 42 new families and review of genetic screening criteria.J Med Genetics.2004;41(7):508-17.

[58]. Tseng CH,Tseng FH Tseng.Diabetes and gastric cancer.The potential risks.World J Gastroenterol.2014;20(7):1701-11.

[59]. ThrumurthySG,ChudryMA,HochhauseD, etal.TheDiaghnosis and management of gastric cancer.Br Med J.2013;347(16):1695-6.

[60]. Crosby DA,DonohoeCL,FitzgeralL, etal.Gastric Neuroendocrine Tumors.Digestive Surg.2004;29(4):331-348.

[61]. Kim J,CheongJH,ChenJ,etal.Menetrier's Diseases in Korea:Report of Two Cases and Review of Cases in a Gastric Cancer Prevalent Region(PDF).YonseiMed J.2004;45(3):555-60.

[62]. Tsukamoto T,MizoshitaT,TatematsuM,etal.Gastric and-intestinal mixed -type intestinal metaplasia:aberrant expression of transcription factors and stem cell intestinalization. Gastric Cancer.2006;9(3):156-66.

[63]. Statistics and outlook for stomach cancer.Cancer Research UK.Retrieved 19 February 2014

[64]. Guidance on Commissioning Cancer Services Improving outcomes un Upper Gastrointestinal Cancers.Arch IranMed.2013;16(6):358-65.

[65]. VirmaniV,KhandelwalA,SethiV,etal.Neoplastic stomach lesions and their mimickers:Spectrum of Imaging manifestations.Cancer Imaging.2012;12:269-78.

[66]. Xu ZQ,BrozaYY,IonsecuR,etal.A nanomaterial-based breath test for distinguishing gastric cancer from benign gastric conditions.BrJ Cancer.2013;108(4):941-50.

[67]. Inoue $\mathrm{H}, \mathrm{KudoS}-$ ShiokawaA,KudoShiokawa.Technologyinsight:laser-scanning confocal microscopy and endoscopy for cellular observation of gastrointestinal tract.NatClinPracGastroenterol\& Hepatol.2005;2(1):31-7.

[68]. Pentenero M, CarrozzoM,PaganoM, et al. Oral acanthosis nigricans,ripe palms and signs of leser-trelat in a patient with gastric adenocarcinoma.Int J Dermatol.2004;43(7):530-2.

[69]. Lim JS,YunMJ,HyungWJ,etal.CT and PET in stomach cancer:preoperative staging and monitoring of response to therapy.Radigraphics.2006;26(1):143-56.

[70]. Detailed Guide:Stomach Cancer Treatment Choices by Type and Stage of StamachCancer.Am Cancer Soc.2009-11-03.

[71]. Guy Slowik(October 2009).What Are The Stages Of Stomach Cancer. Chealthmd.com.

[72]. Detailed Guide:StomachCancer:How Is Stomach Cancer Stage.Am Cancer Soc.

[73]. ChongruksutWilaiwan,LimpakanSirikan(Yamada),BandhuphatChakrabandhu,etal.Correlation of Helicobacter pylori and interleukin-8 mRNA expression in high risk gastric cancer population presdiction. Worl J Gastroenterol Oncol.2006;8(2):215-21.

[74]. RoopmaWadhwa,TakashiTaketa,KazukiSudo,etal.Modern Oncological Approaches to Gastric Adenocarcinoma.GasteroenterolClinNorth Am.20123;42(2):359-69.

[75]. KeChen,Xiao-Wu,Ren-Chao Zhang,etal.Systematic review ans meta-analysis of laproscopy-assisted and open total gastrectomy for gastric cancer.World J Gastroenterol.2013;19(32):5365-76.

[76]. Jennifer L,Pertz Jennifer Y,Wo Harvey J,etal.ChemoradiationTherapy:Localized Esophageal Gastric and Pancreatic Cancer.SurgOncolClin North Am. 2011; 22(3): 511-24.

[77]. Judith Meza-Junco,Heather-Jane Au,Micheal B Sawyer,etal.Critical appraisal of trastuzumb in treatment of advanced cancer.CancerManagement and Research. 2011; 2011(3):57-64.

[78]. Best LM,MughalM,GurusamyKS,Laproscopic versus open gastrectomy for gastric cancer.The Cochrane database of systematic reviews.2016;3:CD011389.

[79]. Sun J,SongY,WangZ,etal.Clinical significance of palliative gastrectomy on the survival of patients with incurable advanced gastric cancer: a systematic review andmeta-analysis.(PDF)BMC Cancer.2013;13(1):577.

[80]. ScartozziM,GaliziaE,VerdeecchiaL,etal.Chemotherapy for advanced gastric cancer :across the years for a standard of care.Expertopnion on Phamacotherapy .2007; 8(6):797-808.

[81]. Fusco N,RoccoEG,Del Conte C,et al.HER2 in gastric cancer: a digital image analysis in pre-neoplastic,primary metastatic lesions.Mod Pathol.2013;26(6):816-24

[82]. CabebeEC,MehtaVK,Fisher G Jr,etal.GastricCancer.Medscape Reference. WebMD. Retrieved 4 April 2014.

[83]. Ford AC,FormanD,HuntRH,etal.Helicobacter pylori eradication therapy to prevent gastric cancer in healthy asymptomatic infected individuals: systematic review and meta-analysis of randomized controlled trials. BMJ.2014;348:g3174.

[84]. Woo HD,Park S,OHK,etal.Diet and cancer risk in the Korean population:a meta-analysis(PDF).Asian Pacific $J$ CancerPreven.2014;15(19):8509-19.

[85]. Kong P,CaiQ,WangJ,etal.Vitamin intake reduce the risk of gastric cancer:meta-analysis and systematic review of randomized and observational studies.PLOS ONE.2014;9(12):e116060.

[86]. BjelakovicG,NikolovaD,SimonettiRG,etal.Antioxidant supplement for preventing gastrointestinal cancers.Cochrane Database ofSystematic Reviews. 2008; (3):CD004183. 